\title{
Risk of post-contrast acute kidney injury in emergency department patients with sepsis
}

\author{
YC Hsu, HY Su, CK Sun, CY Liang, TB Chen, CW Hsu *
}

\section{A B S T R A C T}

Introduction: Although computed tomography (CT) is a useful tool for exploring occult infection in patients with sepsis in the emergency department, the potential nephrotoxicity of contrast media is a major concern. Our study aimed to investigate the association between use of contrast-enhanced $\mathrm{CT}$ and the risks of acute kidney injury and other adverse outcomes in patients with sepsis.

Methods: In total, 587 patients with sepsis who underwent CT scan (enhanced CT group: 105, non-enhanced CT group: 482) from January 2012 to December 2016 at a tertiary referral centre were enrolled in this retrospective analysis, and propensity score matching was performed to minimise the selection bias. The length of stay, incidences of acute kidney injury and emergent dialysis, and short-term mortality were compared between the two groups.

Results: Compared with patients in the nonenhanced CT group, patients in the contrastenhanced CT group did not have increased risks of acute kidney injury (odds ratio $[\mathrm{OR}]=1.38,95 \%$ confidence interval $[\mathrm{CI}]=0.55-3.43 ; \quad \mathrm{P}=0.489$ ), emergent dialysis $(\mathrm{OR}=1.31,95 \% \mathrm{CI}=0.47-3.68$; $\mathrm{P}=0.602)$, or short-term mortality $(\mathrm{OR}=0.90,95 \%$

This article was published on 4 Dec 2019 at www.hkmj.org. $\mathrm{CI}=0.48-1.69 ; \mathrm{P}=0.751)$. In addition, there was no significant difference in the median length of

(20 vs 19 days, $\mathrm{P}=0.742$ ).

Conclusions: Intravenous contrast administration during CT scanning was not associated with prolonged length of hospital stay in patients with sepsis in an emergency setting. Moreover, the use of contrast-enhanced CT was not associated with increased risks of acute kidney injury, emergent dialysis, or short-term mortality.

Hong Kong Med J 2019;25:429-37

https://doi.org/10.12809/hkmj198086
YC Hsu, MD
${ }^{1} \mathrm{HY} \mathrm{Su}, \mathrm{MD}$
${ }_{1,2}$ CK Sun, MD, PhD
${ }^{3,4} \mathrm{CY}$ Liang, $\mathrm{MD}$
${ }^{5}$ TB Chen, PhD
1,2 CW Hsu *, MD, PhD
Department of Emergency Medicine, E-Da Hospital, I-Shou University, Kaohsiung, Taiwan
School of Medicine for International Students, College of Medicine, I-Shou University, Kaohsiung, Taiwan
${ }_{3}^{3}$ Department of Emergency Medicine, E-Da Cancer Hospital, I-Shou University, Kaohsiung, Taiwan
${ }^{4}$ Department of Information Engineering, I-Shou University, Kaohsiung, Taiwan
${ }^{5}$ Department of Medical Imaging and Radiological Sciences, I-Shou University, Kaohsiung, Taiwan

* Corresponding author: saab30002000@gmail.com

New knowledge added by this study

- The risks of nephrotoxicity and other adverse outcomes (ie, emergent dialysis, short-term mortality, and increased length of stay) were not increased after intravenous contrast administration during computed tomography scanning of patients with sepsis.

- Renal function improved within 48-72 hours after computed tomography scans, relative to initial measurements in all patients, suggesting that sepsis (not the administration of contrast media) was the primary determinant of clinical outcomes.

Implications for clinical practice or policy

- The lack of a significant correlation between the administration of contrast agents and the risk of acute kidney injury in patients with sepsis conflicts with the tendency to withhold contrast-enhanced computed tomography for the diagnostic assessment and management of sepsis in the emergency setting.

- After weighing the benefits and risks of contrast administration, clinicians could utilise contrast-enhanced computed tomography scanning in a reasonable manner in critically ill patients with sepsis, in order to identify occult infection foci earlier and facilitate prompt medical management.

\section{Background}

Sepsis is a life-threatening condition that contributes to nearly 850000 emergency department (ED) visits annually in the US. ${ }^{1}$ According to the practice guidelines published by the Surviving Sepsis
Campaign, a care bundle of sepsis treatmentincluding fluid resuscitation, antimicrobial therapy, and source control-is recommended as life-saving treatment for patients with sepsis. ${ }^{2}$ Computed tomography $(\mathrm{CT})$ scanning is a popular method for 


\section{顯影劑造成急診敗血症病人發生急性腎衰竭的 風險}

徐英洲、蘇泓源、孫灼均、梁志宇、陳泰賓、許智偉

引言：雖然電腦斷層顯影是診斷急診敗血症病人感染源的利器, 但是 顯影劑可能產生急性腎衰竭的憂慮, 會使得臨床醫師不敢使用, 進而 無法確定病人診斷。本研究旨在探討急診敗血症病人注射顯影劑是否 跟急性腎衰竭有相關性以及造成敗血症病人產生急性腎衰竭與其他嚴 重後果的風險因子。

方法：自2012年1月至2016年12月，我們收集急診587位有進行電腦 斷層掃描的敗血症病人進行回溯性研究。其中注射顯影劑病人有 105 人, 無注射顯影劑者 482 人。為了降低選擇誤差的干擾, 我們使用傾 向評分匹配來比較兩組之間的住院天數、急性腎衰竭與緊急洗腎發生 率和死亡率。

結果：與無注射顯影劑患者相比, 注射顯影劑患者的急性腎衰竭 （比值比 $=1.38 ， 95 \%$ 置信區間=0.55-3.43；P=0.489）、緊急洗腎 （比值比 $=1.31 ， 95 \%$ 置信區間 $=0.47-3.68 ; \mathrm{P}=0.602$ ) 或短期死亡 率（比值比 $=0.90 ， 95 \%$ 置信區間 $=0.48-1.69 ; \mathrm{P}=0.751)$ 風險均沒 有增加。兩組倖存者間的住院時間中位數亦無顯著差異 (20天對 19 天, $\mathrm{P}=0.742$ )

結論：有進行電腦斷層掃描的急診敗血症病人中, 有注射顯影劑組跟 無注射顯影幾組進行比較, 不管是急性腎衰竭、緊急洗腎、住院天數 和死亡率的風險並無不同。 identifying the focus of infection and guiding the implementation of an appropriate antimicrobial strategy in emergency medical care settings. ${ }^{3}$ The utilisation of CT scans in the ED has increased considerably, such that more than 70 million CT scans are performed in the US annually. ${ }^{4}$ Approximately one in seven patients undergoes a $\mathrm{CT}$ scan during evaluation in the ED. ${ }^{5}$

Although the use of iodinated contrast media is an important method for improving the diagnostic accuracy of CT examination, ${ }^{6}$ there are concerns regarding the potential for precipitating renal dysfunction, especially in patients who already have impaired renal function. ${ }^{7,8}$ The third leading cause of acute kidney injury (AKI) in hospitalised patients is reported to be contrast-associated (CA)-AKI ${ }^{9}$; CA-AKI is associated with increased risks of major adverse events, including myocardial infarction, renal failure, and mortality. ${ }^{7,10}$ Nevertheless, it remains controversial whether an association exists between intravenous administration of contrast media during $\mathrm{CT}$ scans and the development of CAAKI. ${ }^{11-13}$ This controversy exists largely because the introduction of refined iso- or low-osmolar contrast agents has reduced the risk of $\mathrm{AKI}^{14}$ and because the majority of previous studies on CA-AKI were performed in patients who underwent coronary angiography, ${ }^{15-17}$ which utilises different dosages and routes of contrast administration relative to those of conventional contrast-enhanced CT scans. ${ }^{18}$ Previous studies on CA-AKI in an emergency setting have been inconclusive. , $7,19-24^{\text {Although }}$ those studies investigated the benefits and risks of contrast administration in many clinical settings, including acute stroke, pulmonary embolism, and trauma, very few of them evaluated the impact of contrast administration on patients with sepsis. Notably, sepsis remains a leading cause of mortality in critically ill patients ${ }^{2}$ and CT imaging studies play important roles in both identifying the source of infection and facilitating infection control in patients with sepsis. Therefore, the aim of the current study was to investigate whether intravenous contrast administration in patients with sepsis is associated with an increased risk of AKI and increased incidences of other adverse clinical outcomes.

\section{Methods}

\section{Study design}

This retrospective cohort study was conducted at a tertiary referral medical centre with approximately 50000 ED visits per year. The study population included all adult (age $\geq 18$ years) patients who visited the ED and underwent $\mathrm{CT}$ scans (including brain, chest, abdomen or extremities) and serial serum creatinine measurements during their initial ED visits and any follow-ups within 48 to 72 hours from 1 January 2012 to 31 December 2016. Patients with sepsis were identified by principal diagnosis and serum lactate measurement, in accordance with Sepsis-3 guidelines. ${ }^{25}$ Patients who received haemodialysis, underwent contrast-enhanced CT scan within 3 months, or experienced a cardiac arrest event before ED arrival were excluded from the analysis. This study protocol followed the STROBE (Strengthening the Reporting of Observational Studies in Epidemiology) guidelines.

\section{Data collection}

Demographic characteristics of the enrolled patients (ie, age and sex) and clinical information (eg, comorbidities, chronic medications, laboratory results, acute illness, types and dosage of contrast agent, and initial and final diagnoses) were obtained from written medical charts and electronic medical records. Co-morbidities were coded based on International Classification of Diseases, Ninth Edition, Clinical Modification diagnostic codes reported in medical records. In accordance with World Health Organization criteria, anaemia was defined as baseline haematocrit values below 39\% and below $36 \%$ for men and women, respectively. ${ }^{26}$ Chronic kidney disease was defined as a baseline estimated glomerular filtration rate (eGFR) $<60$ $\mathrm{mL} / \mathrm{min} / 1.73 \mathrm{~m}^{2}$, calculated using the Modification of Diet in Renal Disease equation. ${ }^{27}$ Baseline renal 
function was calculated according to each patient's serum creatinine level at 24 hours before the CT scan. The presence of shock was identified by the need for vasopressors to maintain haemodynamic stability despite adequate fluid administration during ED stay.

\section{Outcome measures}

We divided the eligible patients for this study into two groups: contrast-enhanced CT and nonenhanced CT; primary and secondary outcomes were recorded and compared between groups. The primary outcome was the incidence of AKI, which was defined as an absolute increase of $0.5 \mathrm{mg} / \mathrm{dL}$ or $>50 \%$ increase in baseline serum creatinine concentration within 48 to 72 hours after CT scan. ${ }^{28}$ The secondary outcomes included the incidences of emergent dialysis (defined as initiation of dialysis during the hospital stay) and short-term mortality (defined as death within 30 days after CT scan), as well as the difference in length of hospital stay for survivors.

\section{Sample size estimation}

The estimation of sample size was performed with PASS 11 software in accordance with the results of previous studies regarding AKI incidence in patients with sepsis $^{29}$ and odds ratio (OR) of CA-AKI. ${ }^{30}$ With a $30 \%$ incidence of AKI in patients with sepsis and an OR of 2.7 for CA-AKI, we determined that 109 patients were needed to detect a significant association with probability (power) of 0.8 and Type 1 error of 0.05 .

\section{Statistical analysis}

Data are presented as means \pm standard deviations or medians with 25th to 75th percentiles (ie, interquartile range) for continuous variables, and as numbers (\%) for categorical variables. Two-sample $t$ tests and Chi squared tests were used to compare continuous and categorical variables, respectively. A two-tailed $\mathrm{P}$ value of $<0.05$ was considered statistically significant. Propensity score matching was performed to reduce potential selection bias and other confounding factors. We calculated the propensity score for each patient by modelling the probability of receiving contrast medium. Variables in the model were composed of factors that influence outcomes related to renal function or influence the selection of contrast medium. We used total 21 variables including age, sex, co-morbidities (ie, diabetes mellitus, hypertension, liver cirrhosis, coronary artery disease, left heart failure, chronic kidney disease, anaemia, chronic obstructive pulmonary disease, dyslipidaemia, and malignancy), nephrotoxic medications (ie, statins, non-steroidal anti-inflammatory drugs, angiotensin-converting enzyme inhibitors or angiotensin II receptor blockers, nephrotoxic antibiotics such as aminoglycosides and vancomycin), laboratory data (ie, initial serum creatinine, eGFR, and serum lactate), measures of illness severity (ie, initial presence of septic shock and need for intensive care unit [ICU] admission) to calculate the propensity scores for all patients. A multivariable logistic regression analysis model using nearest-neighbour matching, calliper 0.1, was generated to predict the probability of receiving contrast medium. We used the resulting propensity scores to match the contrast-enhanced CT group members with non-enhanced CT group members at a ratio of $1: 1$. Patients without a corresponding match were excluded. All statistical analyses were performed using SPSS (Windows version 22.0; IBM Corp, Armonk [NY], US).

\section{Results}

\section{Study population and contrast agents}

During the study period, 200427 adult patients visited the ED; of these, 712 met the criteria for inclusion in this study. After further exclusion of patients with elevated serum lactate levels from shock with non-septic aetiology, the remaining 587 patients (enhanced CT group: 105; nonenhanced CT group: 482) were analysed (Fig). In the contrast-enhanced CT group, 23 patients received intravenous iopromide (Ultravist 370; Bayer Parma AG, Berlin, Germany) and 82 patients received intravenous iohexol (Omnipaque; Bayer Parma AG, Berlin, Germany). Only one patient received a contrast volume $>100 \mathrm{~mL}(120 \mathrm{~mL})$.

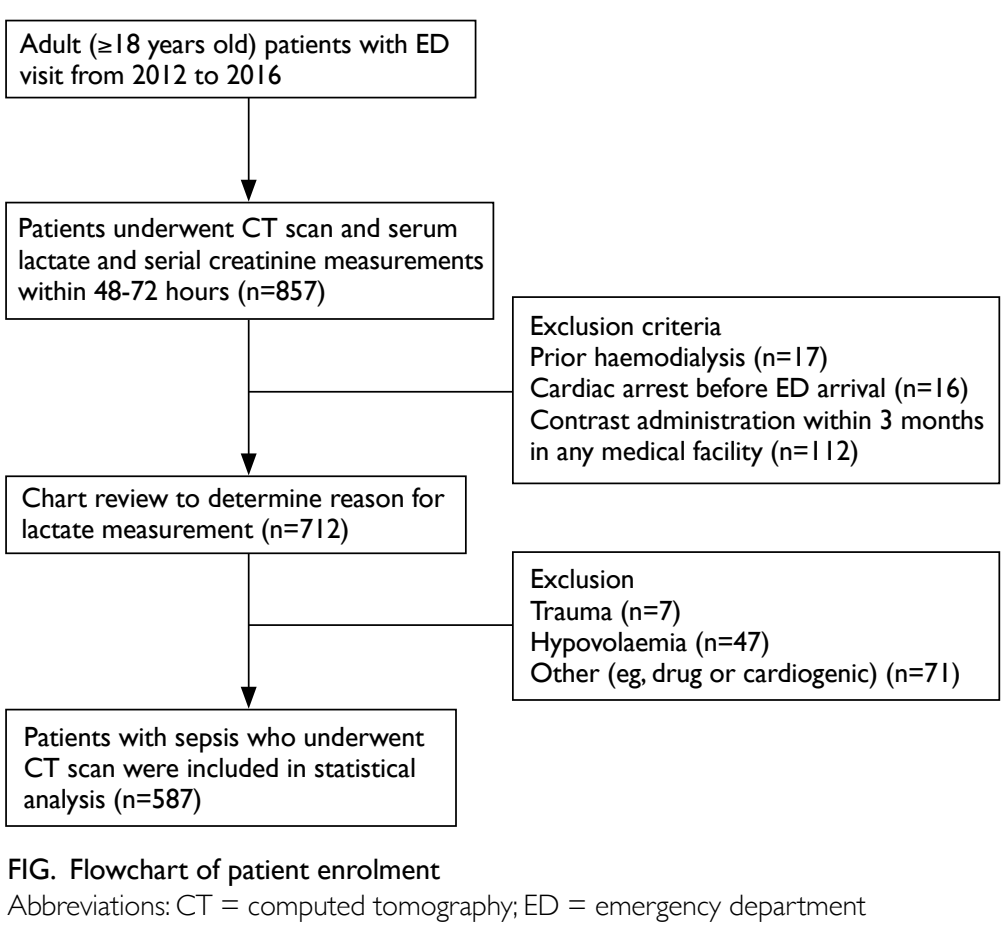


Prior to propensity score matching, patients in initial and follow-up serum creatinine levels, and the contrast-enhanced CT group were significantly had higher initial serum lactate levels than those younger; moreover, they had lower prevalences of in the non-enhanced CT group. There were no hypertension, chronic kidney disease, and chronic significant differences in the incidences of shock and obstructive pulmonary disease, compared to ICU admission between the two groups (Table 1). patients in the non-enhanced CT group. Patients in the enhanced CT group also had significantly lower

TABLE I. Demographic and characteristics of patients with sepsis $(n=587)^{*}$

\begin{tabular}{|c|c|c|c|}
\hline & Enhanced CT $(n=105)$ & $\begin{array}{c}\text { Non-enhanced CT } \\
(\mathrm{n}=482)\end{array}$ & $\mathbf{P}$ value \\
\hline \multicolumn{4}{|l|}{ Characteristics } \\
\hline Age $(y)$ & $64.3 \pm 14.5$ & $69.9 \pm 15.6$ & $<0.001$ \\
\hline Male & $63(60.0 \%)$ & $288(59.8 \%)$ & 0.962 \\
\hline \multicolumn{4}{|l|}{ Co-morbiditiesł } \\
\hline Hypertension & $52(49.5 \%)$ & $293(60.8 \%)$ & 0.034 \\
\hline Diabetes mellitus & $54(51.4 \%)$ & $227(47.1 \%)$ & 0.421 \\
\hline Liver cirrhosis & $12(11.4 \%)$ & $48(10.0 \%)$ & 0.652 \\
\hline Left heart failure & $13(12.4 \%)$ & $66(13.7 \%)$ & 0.721 \\
\hline Coronary artery disease & $9(8.6 \%)$ & $67(13.9 \%)$ & 0.141 \\
\hline Chronic kidney disease§ & $45(42.9 \%)$ & $298(61.8 \%)$ & $<0.001$ \\
\hline Anaemiall & $65(61.9 \%)$ & $339(70.3 \%)$ & 0.091 \\
\hline Chronic obstructive pulmonary disease & $3(2.9 \%)$ & $48(10.0 \%)$ & 0.019 \\
\hline Dyslipidaemia & $15(14.3 \%)$ & $57(11.8 \%)$ & 0.486 \\
\hline Malignancy & $15(14.3 \%)$ & $89(18.5 \%)$ & 0.309 \\
\hline \multicolumn{4}{|l|}{ Nephrotoxic medication } \\
\hline Statins & $7(6.7 \%)$ & $48(10.0 \%)$ & 0.294 \\
\hline NSAIDs & $40(38.1 \%)$ & $154(32.0 \%)$ & 0.225 \\
\hline Nephrotoxic antibiotics & $5(4.8 \%)$ & $29(6.0 \%)$ & 0.618 \\
\hline ACEIs/ARBs & $15(14.3 \%)$ & $95(19.7 \%)$ & 0.197 \\
\hline Contrast volume $>100 \mathrm{~mL}$ & $1(0.9 \%)$ & - & \\
\hline \multicolumn{4}{|l|}{ Laboratory result } \\
\hline Initial SCr (mg/dL) & $1.5(1.1-1.9)$ & $2.0(1.3-3.1)$ & 0.028 \\
\hline Initial eGFR (mL/min/1.73 m²) & $44(33-65)$ & $32(19-52)$ & 0.048 \\
\hline $\mathrm{sCr}$ at $48-72 \mathrm{~h}(\mathrm{mg} / \mathrm{dL})$ & $1.2(0.9-1.7)$ & $1.4(1.0-2.5)$ & $<0.001$ \\
\hline eGFR at $48-72 \mathrm{~h}\left(\mathrm{~mL} / \mathrm{min} / 1.73 \mathrm{~m}^{2}\right)$ & $58(37-84)$ & $49(25-73)$ & $<0.001$ \\
\hline Initial lactate (mmoL/L) & $2.60(1.41-4.49)$ & $2.22(1.39-4.09)$ & 0.004 \\
\hline \multicolumn{4}{|l|}{ Acute illness } \\
\hline Shock $^{\star *}$ & $52(49.5 \%)$ & $239(49.6 \%)$ & 0.991 \\
\hline ICU admission & 70 (66.7\%) & 325 (67.4\%) & 0.880 \\
\hline
\end{tabular}

Abbreviations: $\mathrm{ACEls}=$ angiotensin-converting enzyme inhibitors; $\mathrm{ARBs}=$ angiotensin $\|$ receptor blockers; $\mathrm{CT}=$ computed tomography; eGFR = estimated glomerular filtration rate; ICU = intensive care unit; NSAIDs = non-steroidal anti-inflammatory drugs; $\mathrm{SCr}=$ serum creatinine

* Data are shown as mean \pm standard deviation, No. (\%) or median (interquartile range), unless otherwise specified

$\dagger$ Chi squared test was used for categorical variable comparison; two-sample $t$ tests were used for continuous variable comparison; $\mathrm{P}<0.05$ was considered to indicate statistical significance

$\ddagger$ Based on International Classification of Diseases, Ninth Edition, Clinical Modification diagnostic codes and admission diagnosis from previous hospitalisation or index emergency department visit

$\S$ Based on the National Kidney Foundation Kidney Disease Outcomes Quality Initiative guideline (baseline eGFR $<60 \mathrm{~mL} / \mathrm{min} /$ $\left.1.73 \mathrm{~m}^{2}\right)$

॥ According to World Health Organization definition: baseline haematocrit $<39 \%$ in men, $<36 \%$ in women

** Requirement of vasopressors during emergency department stay 
CT group were successfully paired with an equal emergent dialysis, and short-term mortality were number of patients in the non-enhanced CT group. not significantly greater in the contrast-enhanced After matching, there were no statistically significant CT group than in the non-enhanced CT group. differences between the two groups in any covariates Five of 44 patients with sepsis in the non-enhanced (Table 2).

CT group who received emergency haemodialysis subsequently required chronic dialysis; however, no

\section{Treatment outcomes}

patients required chronic dialysis in the contrast-

Before propensity score matching, the risks of AKI, enhanced CT group. Furthermore, there was no

TABLE 2. Demographic and characteristics of matched patients with sepsis $(n=202)^{*}$

\begin{tabular}{|c|c|c|c|}
\hline & Enhanced CT $(n=101)$ & $\begin{array}{c}\text { Non-enhanced CT } \\
(n=101)\end{array}$ & $P$ valuet \\
\hline \multicolumn{4}{|l|}{ Characteristics } \\
\hline Age (y) & $64.4 \pm 14.6$ & $66.4 \pm 16.6$ & 0.354 \\
\hline Male & $63(62.4 \%)$ & $63(62.4 \%)$ & 1.000 \\
\hline \multicolumn{4}{|l|}{ Co-morbidities $\ddagger$} \\
\hline Hypertension & $50(49.5 \%)$ & $53(52.5 \%)$ & 0.673 \\
\hline Diabetes mellitus & $50(49.5 \%)$ & $50(49.5 \%)$ & 1.000 \\
\hline Liver cirrhosis & $12(11.9 \%)$ & $15(14.9 \%)$ & 0.535 \\
\hline Left heart failure & $13(12.9 \%)$ & $18(17.8 \%)$ & 0.329 \\
\hline Coronary artery disease & $9(8.9 \%)$ & $12(11.9 \%)$ & 0.489 \\
\hline Chronic kidney disease§ & $44(43.6 \%)$ & $45(44.6 \%)$ & 0.887 \\
\hline Anaemiall & $64(63.4 \%)$ & $65(64.4 \%)$ & 0.884 \\
\hline Chronic obstructive pulmonary disease & $3(3.0 \%)$ & $5(5.0 \%)$ & 0.481 \\
\hline Dyslipidaemia & $12(11.9 \%)$ & $14(13.9 \%)$ & 0.674 \\
\hline Malignancy & $15(14.9 \%)$ & $14(13.9 \%)$ & 0.841 \\
\hline \multicolumn{4}{|l|}{ Nephrotoxic medication } \\
\hline Statins & 7 (6.9\%) & $8(7.9 \%)$ & 0.294 \\
\hline NSAIDs & $37(36.6 \%)$ & $41(40.6 \%)$ & 0.563 \\
\hline Nephrotoxic antibiotics & $4(4.0 \%)$ & $6(5.9 \%)$ & 0.517 \\
\hline ACEIs/ARBs & $15(14.9 \%)$ & $15(14.9 \%)$ & 1.000 \\
\hline \multicolumn{4}{|l|}{ Laboratory result } \\
\hline Initial SCr (mg/dL) & $1.5(1.1-1.9)$ & $14(1.1-2.1)$ & 0.808 \\
\hline Initial eGFR (mL/min/1.73 m²) & $44(33-65)$ & $46(33-68)$ & 0.799 \\
\hline $\mathrm{sCr}$ at $48-72 \mathrm{~h}(\mathrm{mg} / \mathrm{dL})$ & $1.2(0.9-1.7)$ & $1.0(0.8-1.6)$ & 0.056 \\
\hline eGFR at $48-72 \mathrm{~h}\left(\mathrm{~mL} / \mathrm{min} / 1.73 \mathrm{~m}^{2}\right)$ & $58(37-82)$ & $67(45-89)$ & 0.084 \\
\hline Initial lactate (mmoL/L) & $2.60(1.35-4.49)$ & $2.44(1.37-4.70)$ & 0.227 \\
\hline \multicolumn{4}{|l|}{ Acute illness } \\
\hline Shock $^{\star \star}$ & $50(49.5 \%)$ & $56(55.4 \%)$ & 0.398 \\
\hline ICU admission & 66 (65.3\%) & $76(75.2 \%)$ & 0.124 \\
\hline
\end{tabular}

Abbreviations: ACEls = angiotensin-converting enzyme inhibitors; $\mathrm{ARBs}=$ angiotensin II receptor blockers; $\mathrm{CT}=$ computed

tomography; eGFR = estimated glomerular filtration rate; ICU = intensive care unit; NSAIDs = non-steroidal anti-inflammatory drugs; $\mathrm{SCr}=$ serum creatinine

* Data are shown as mean \pm standard deviation, No. (\%) or median (interquartile range), unless otherwise specified

+ Chi squared test was used for categorical variable comparison; two-sample $t$ tests were used for continuous variable comparison; $\mathrm{P}<0.05$ was considered to indicate statistical significance

‡ Based on International Classification of Diseases, Ninth Edition, Clinical Modification diagnostic codes and admission diagnosis from previous hospitalisation or index emergency department visit

$\S \quad$ Based on the National Kidney Foundation Kidney Disease Outcomes Quality Initiative guideline (baseline eGFR <60 mL/min/ $1.73 \mathrm{~m}^{2}$ )

॥ According to World Health Organization definition: baseline haematocrit $<39 \%$ in men, $<36 \%$ in women

* Requirement of vasopressors during emergency department stay 
significant difference in the length of hospital stay between the two groups (Table 3 ). The same results were observed after propensity score matching: there were no notable differences in the risks of AKI, emergent dialysis, or short-term mortality; the median length of hospital stay was also similar between the matched contrast-enhanced and nonenhanced CT groups (Table 4).

\section{Discussion}

In this ED-based single-centre retrospective study, we performed a subgroup analysis to investigate the possible adverse clinical impacts of contrast agent administration in patients with sepsis. By using propensity score matching, we demonstrated that intravenous administration of contrast media in patients with sepsis was not associated with increased risks of AKI or other adverse outcomes, following contrast-enhanced CT scans to identify foci of infection. During revision of this manuscript, Hinson et $\mathrm{al}^{31}$ reported a retrospective cohort study; they concluded that contrast medium administration was not associated with increased incidence of AKI in patients with sepsis, consistent with our findings. Compared with the study by Hinson et al, the patients in our study had more severe sepsis (ie, higher incidences of shock and ICU admission); moreover, our findings revealed that administration of reasonable volumes of contrast medium did not increase the risks of emergency dialysis or short-term mortality. Thus, clinicians can use contrast-enhanced CT scans in a reasonable manner in septic patients, in order to identify occult infection foci earlier and facilitate prompt medical management.

Our patients had surprisingly high prevalences of hypertension, diabetes mellitus, and chronic kidney disease, which could have been related to their older age, as reported in a prior study. ${ }^{32}$ Before propensity score matching, patients in the contrast-enhanced CT group were significantly younger and had fewer co-morbidities, including hypertension, chronic kidney disease, and chronic obstructive pulmonary disease. Moreover, patients in the contrast-enhanced CT group had lower

TABLE 3. Outcomes analysis of unmatched patients with sepsis

\begin{tabular}{|c|c|c|c|c|}
\hline & $\begin{array}{c}\text { Enhanced CT } \\
(n=105)\end{array}$ & $\begin{array}{c}\text { Non-enhanced CT } \\
(n=482)\end{array}$ & Odds ratio* $(95 \% \mathrm{Cl})$ & $P$ value \\
\hline Acute kidney injury† & $13(12.4 \%)$ & $51(10.6 \%)$ & $1.19(0.62-2.29)$ & 0.592 \\
\hline Emergent dialysis $\ddagger$ & $11(10.5 \%)$ & $44(9.1 \%)$ & $1.17(0.58-2.34)$ & 0.668 \\
\hline \multirow[t]{2}{*}{ Short-term mortality§ } & $27(25.7 \%)$ & $128(26.6 \%)$ & $0.96(0.59-1.55)$ & 0.859 \\
\hline & Enhanced CT $(n=77)$ & $\begin{array}{l}\text { Non-enhanced CT } \\
\qquad(n=356)\end{array}$ & & $P$ value \\
\hline Length of stay (median [IQR], days)॥ & $19(10-32)$ & $17(10-30)$ & & 0.419 \\
\hline \multicolumn{5}{|c|}{$\begin{array}{l}\text { Abbreviations: } \mathrm{Cl}=\text { confidence interval; } \mathrm{CT}=\text { computed tomography; IQR = interquartile range } \\
* \text { Odds of enhanced CT group vs unenhanced CT group } \\
+ \text { Acute kidney injury: defined as baseline creatinine }>0.5 \mathrm{mg} / \mathrm{dL} \text { or } 50 \% \text { increase within } 48-72 \text { hours after CT scan } \\
\text { † Emergent dialysis: initiation of dialysis during hospital stay } \\
\S \text { Short-term mortality: death within } 30 \text { days after CT scan } \\
\| \text { Length of stay: including intensive care unit and ward stays, excluding patients with mortality }\end{array}$} \\
\hline
\end{tabular}

TABLE 4. Outcomes analysis of matched patients with sepsis

\begin{tabular}{|c|c|c|c|c|}
\hline & $\begin{array}{c}\text { Enhanced CT } \\
\qquad(n=101)\end{array}$ & $\begin{array}{c}\text { Non-enhanced CT } \\
(n=101)\end{array}$ & Odds ratio* (95\% Cl) & $P$ value \\
\hline Acute kidney injury† & $12(11.9 \%)$ & $9(8.9 \%)$ & $1.38(0.55-3.43)$ & 0.489 \\
\hline Emergent dialysis $\ddagger$ & $9(8.9 \%)$ & $7(6.9 \%)$ & $1.31(0.47-3.68)$ & 0.602 \\
\hline \multirow[t]{2}{*}{ Short-term mortality§ } & $26(25.7 \%)$ & $28(27.7 \%)$ & $0.90(0.48-1.69)$ & 0.751 \\
\hline & Enhanced CT $(n=74)$ & $\begin{array}{l}\text { Non-enhanced CT } \\
(\mathrm{n}=72)\end{array}$ & & $P$ value \\
\hline Length of stay (median [IQR], days)॥ & $20(10-31)$ & $19(9-33)$ & & 0.742 \\
\hline
\end{tabular}

Abbreviations: $\mathrm{Cl}=$ confidence interval; $\mathrm{CT}=$ computed tomography; $\mathrm{IQR}=$ interquartile range

* Odds of enhanced CT group vs unenhanced CT group

$\dagger$ Acute kidney injury: defined as baseline creatinine $>0.5 \mathrm{mg} / \mathrm{dL}$ or $50 \%$ increase within $48-72$ hours after CT scan

‡ Emergent dialysis: initiation of dialysis during hospital stay

$\S$ Short-term mortality: death within 30 days after CT scan

$\|$ Length of stay: including intensive care unit and ward stays, excluding patients with mortality 
initial serum creatinine levels and higher eGFRs, as observed in other studies. ${ }^{6,32}$ This could be related to the common clinical practice of using contrastenhanced CT for younger patients with few comorbidities and relatively good renal function, based on considerations of the potential nephrotoxicities of the contrast agents; ; a few patients with poor renal function (24 of 482 patients with sepsis in the non-enhanced CT group) may also have avoided contrast agents following an explanation of the potential for nephrotoxicity. Clinicians may have hesitated to administer contrast media to patients with respiratory disease because of the risk of immediate hypersensitivity reaction; however, asthma and chronic obstructive pulmonary disease have not been established as consistent risk factors for contrast media-related adverse drug reactions. ${ }^{33}$ The lack of significant differences in risks of AKI, emergent dialysis, and short-term mortality between the non-enhanced and enhanced CT groups before propensity score matching in our study may have been influenced by the above-mentioned tendency for clinicians to perform contrast-enhanced CT in presumably healthier patients. However, it is difficult to evaluate the causal relationship between administration of contrast agents and risk of AKI in patients with sepsis by comparing two patient groups with many different demographic and characteristics; therefore, we used propensity score matching to minimise the impacts of potential confounders.

Although the mean initial serum lactate level in the contrast-enhanced CT group was slightly but significantly higher than that in the non-enhanced CT group, this difference was not correlated with the incidences of acute illness (eg, shock), ICU admission, and short-term mortality between the two groups. In addition, the levels of renal function, reflected by serum creatinine levels and eGFRs within 48 to 72 hours after CT scans, improved relative to initial measurements in both groups. These seemingly paradoxical findings suggested that sepsis, rather than the administration of contrast media, was the determinant of clinical outcomes in the present study.

Previous studies in emergency medical settings have shown wide variation in the incidence of postcontrast AKI (3.2\%-12\%); this may be partially explained by the variety of diseases encountered in the ED, as well as differences in the definitions of AKI adopted in each study. ${ }^{6,719-24,31}$ Nearly half of the patients (49\%) in the present study experienced septic shock; thus, the increased incidence of postcontrast AKI in our patients (12.4\%), compared with that observed in prior studies, may be attributed to the impaired physical status of our patients. This may also explain the considerably higher rates of emergent dialysis and short-term mortality, as well as the increased median length of hospital stay for survivors among our patients, compared to those parameters measured in other studies that did not focus on patients with sepsis. ${ }^{21,34}$

Thus far, the pathophysiology of CA-AKI remains poorly characterised. Based on the results of some animal studies, proposed mechanisms include acute tubular necrosis caused by medullary hypoxia from vasoconstriction, as well as direct cytotoxic effects of the contrast agent on renal tubular cells. ${ }^{35,36}$ Compared with AKI caused by other aetiologies, CA-AKI involves relatively rapid recovery of renal function; this is potentially because of the reduced extent of tubular necrosis, which leads to minor and transient functional impairment of tubular epithelial cells. ${ }^{37}$ Nevertheless, sepsis is the leading cause of AKI in critically ill patients and is associated with a higher mortality rate among patients in the ICU, compared with patients who have AKI caused by other aetiologies..$^{38}$ Therefore, hesitation to perform contrast-enhanced CT scans for patients with sepsis, in order to identify occult infection foci, could result in delayed diagnoses of life-threatening conditions that carry considerable risks of morbidity and mortality, even in patients with serum creatinine up to $4.0 \mathrm{mg} / \mathrm{dL} .^{6}$

A number of studies performed in the past several years have been designed to maintain a balance between the benefits and adverse effects of contrastenhanced CT scans in many clinical settings. ${ }^{6,7,12,20-22}$ The vast majority of those studies showed no significant association between the use of contrast agents and an increased risk of AKI. Consistent with the prior findings, contrast-enhanced CT scans of our patients with sepsis were not associated with increased risks of AKI and other adverse clinical outcomes. Among all aetiologies of AKI in patients requiring emergent medical attention, such as sepsis, dehydration, and nephrotoxic medication use, ${ }^{18}$ the contribution of CA-AKI is regarded as considerably less important ${ }^{37}$; notably, our findings support this view. Furthermore, it has been consistently shown that the performance of a contrast-enhanced CT scan is justified in patients for whom the examination is indicated, provided that other risk factors of AKI are well controlled. ${ }^{39}$

There are several limitations in our study, largely in relation to its single-centre and retrospective design. First, the non-enhanced CT group consisted of older patients with a higher prevalence of hypertension and worse renal function; this suggested a selection bias. Although we routinely checked serum lactate for patients with suspected sepsis in the ED, there were a few patients diagnosed with sepsis who did not have lactate measurement data; this may also have resulted in selection bias. Second, although propensity score matching was used to minimise the impacts of 
potential confounders, unmeasured confounding variables remained, leading to potentially biased results. Therefore, further large-scale cohort or well-controlled prospective randomised studies are warranted. Finally, the definition of AKI used in this study (elevation of serum creatinine concentration by $0.5 \mathrm{mg} / \mathrm{dL}$ or by $50 \%$ increase relative to baseline within 48 to 72 hours after contrast administration) may not accurately reflect the clinical condition because the relationship between increases in serum creatinine level and deterioration of renal function is reportedly non-linear. ${ }^{40}$

\section{Conclusion}

Our study demonstrated that the intravenous administration of contrast media during $\mathrm{CT}$ scans was not associated with increased risks of AKI, emergent dialysis, or short-term mortality for patients with sepsis in the ED; moreover, the use of contrast-enhanced CT was not associated with prolonged length of hospital stay in these patients. The lack of a significant correlation between the administration of contrast agents and the risk of AKI in patients with sepsis conflicts with the tendency to withhold contrast-enhanced CT for the diagnostic assessment and management of sepsis in the emergency setting. Further studies are necessary to confirm these findings and provide further guidance for clinical practice.

\section{Author contributions}

All authors had full access to the data, contributed to the study, approved the final version for publication, and take responsibility for its accuracy and integrity.

Concept or design: YC Hsu.

Acquisition of data: $\mathrm{HY} \mathrm{Su}, \mathrm{CW}$ Hsu, CY Liang.

Analysis or interpretation of data: TB Chen.

Drafting of the article: YC Hsu.

Critical revision for important intellectual content: CK Sun, CW Hsu.

\section{Acknowledgement}

Dr Chi-feng Hsieh is acknowledged for providing technical support in sample size calculation.

\section{Conflicts of interest}

All authors have disclosed no conflicts of interest.

\section{Funding/support}

This research received no specific grant from any funding agency in the public, commercial, or not-for-profit sectors.

\section{Ethics approval}

The study was approved by the Institutional Review Board of E-Da hospital (EMRP-106-037) and the requirement for informed patient consent was waived because of the retrospective observational nature of the study.

\section{References}

1. Wang HE, Jones AR, Donnelly JP. Revised national estimates of emergency department visits for sepsis in the United States. Crit Care Med 2017;45:1443-9.

2. Rhodes A, Evans LE, Alhazzani W, et al. Surviving Sepsis Campaign: international guidelines for management of sepsis and septic shock: 2016. Intensive Care Med 2017;43:304-77.

3. Jimenez MF, Marshall JC; International Sepsis Forum. Source control in the management of sepsis. Intensive Care Med 2001;27 Suppl 1:S49-62.

4. Berrington de González A, Mahesh M, Kim KP, et al. Projected cancer risks from computed tomographic scans performed in the United States in 2007. Arch Intern Med 2009;169:2071-7.

5. Kocher KE, Meurer WJ, Fazel R, Scott PA, Krumholz HM, Nallamothu BK. National trends in use of computed tomography in the emergency department. Ann Emerg Med 2011;58:452-62.e3.

6. Hinson JS, Ehmann MR, Fine DM, et al. Risk of acute kidney injury after intravenous contrast media administration. Ann Emerg Med 2017;69:577-86.e4.

7. Mitchell AM, Kline JA, Jones AE, Tumlin JA. Major adverse events one year after acute kidney injury after contrast-enhanced computed tomography. Ann Emerg Med 2015;66:267-74.e4.

8. McCullough PA, Adam A, Becker CR, et al. Epidemiology and prognostic implications of contrast-induced nephropathy. Am J Cardiol 2006;98:5K-13K.

9. Nash K, Hafeez A, Hou S. Hospital-acquired renal insufficiency. Am J Kidney Dis 2002;39:930-6.

10. Coca SG, Peixoto AJ, Garg AX, Krumholz HM, Parikh CR. The prognostic importance of a small acute decrement in kidney function in hospitalized patients: a systematic review and meta-analysis. Am J Kidney Dis 2007;50:71220 .

11. Katzberg RW, Newhouse JH. Intravenous contrast medium-induced nephrotoxicity: is the medical risk really as great as we have come to believe? Radiology 2010;256:218.

12. McDonald JS, McDonald RJ, Comin J, et al. Frequency of acute kidney injury following intravenous contrast medium administration: a systematic review and metaanalysis. Radiology 2013;267:119-28.

13. Kashani K, Levin A, Schetz M. Contrast-associated acute kidney injury is a myth: We are not sure. Intensive Care Med 2018;44:110-4.

14. Lameire N, Kellum JA; KDIGO AKI Guideline Work Group. Contrast-induced acute kidney injury and renal support for acute kidney injury: a KDIGO summary (Part 2). Crit Care 2013;17:205

15. Mehran R, Aymong ED, Nikolsky E, et al. A simple risk score for prediction of contrast-induced nephropathy after percutaneous coronary intervention: development and initial validation. J Am Coll Cardiol 2004;44:1393-9.

16. Mehran R, Nikolsky E. Contrast-induced nephropathy: definition, epidemiology, and patients at risk. Kidney Int Suppl 2006;(100):S11-5.

17. Rihal CS, Textor SC, Grill DE, et al. Incidence and prognostic importance of acute renal failure after percutaneous coronary intervention. Circulation 2002;105:2259-64.

18. Aycock RD, Westafer LM, Boxen JL, Majlesi N, Schoenfeld EM, Bannuru RR. Acute kidney injury after computed 
tomography: a meta-analysis. Ann Emerg Med 2018;71:4453.e4.

19. Mitchell AM, Kline JA. Contrast nephropathy following computed tomography angiography of the chest for pulmonary embolism in the emergency department. J Thromb Haemost 2007;5:50-4.

20. Sonhaye L, Kolou B, Tchaou M, et al. Intravenous contrast medium administration for computed tomography scan in emergency: a possible cause of contrast-induced nephropathy. Radiol Res Pract 2015;2015:805786.

21. Heller M, Krieger P, Finefrock D, Nguyen T, Akhtar S. Contrast CT scans in the emergency department do not increase risk of adverse renal outcomes. West J Emerg Med 2016;17:404-8

22. Ehrlich ME, Turner HL, Currie LJ, Wintermark M, Worrall BB, Southerland AM. Safety of computed tomographic angiography in the evaluation of patients with acute stroke: a single-center experience. Stroke 2016;47:2045-50.

23. Lima FO, Lev MH, Levy RA, et al. Functional contrastenhanced CT for evaluation of acute ischemic stroke does not increase the risk of contrast-induced nephropathy. AJNR Am J Neuroradiol 2010;31:817-21.

24. Tremblay LN, Tien H, Hamilton P, et al. Risk and benefit of intravenous contrast in trauma patients with an elevated serum creatinine. J Trauma 2005;59:1162-6.

25. Singer M, Deutschman CS, Seymour CW, et al. The third international consensus definitions for sepsis and septic shock (Sepsis-3). JAMA 2016;315:801-10.

26. World Health Organ Tech Rep Ser. Nutritional anaemias: report of a WHO scientific group. World Health Organ Tech Rep Ser 1968;405:5-37.

27. National Kidney Foundation. K/DOQI clinical practice guidelines for chronic kidney disease: evaluation, classification, and stratification. Am J Kidney Dis 2002;39:S1-266.

28. Mehta RL, Kellum JA, Shah SV, et al. Acute Kidney Injury Network: report of an initiative to improve outcomes in acute kidney injury. Crit Care 2007;11:R31.
29. Bagshaw SM, George C, Bellomo R; ANZICS Database Management Committee. Early acute kidney injury and sepsis: a multicentre evaluation. Crit Care 2008;12:R47.

30. Song W, Zhang T, Pu J, Shen L, He B. Incidence and risk of developing contrast-induced acute kidney injury following intravascular contrast administration in elderly patients. Clin Interv Aging 2014;9:85-93.

31. Hinson JS, Al Jalbout N, Ehmann MR, Klein EY. Acute kidney injury following contrast media administration in the septic patient: A retrospective propensity-matched analysis. J Crit Care 2019;51:111-6.

32. McDonald, JS, McDonald RJ, Williamson EE, Kallmes DF, Kashani K. Post-contrast acute kidney injury in intensive care unit patients: a propensity score-adjusted study. Intensive Care Med 2017;43:774-84.

33. Bettmann MA, Heeren T, Greenfield A, Goudey C. Adverse events with radiographic contrast agents: results of the SCVIR Contrast Agent Registry. Radiology 1997;203:61120.

34. McDonald RJ, McDonald JS, Carter RE, et al. Intravenous contrast material exposure is not an independent risk factor for dialysis or mortality. Radiology 2014;273:714-25.

35. Persson PB, Hansell P, Liss P. Pathophysiology of contrast medium-induced nephropathy. Kidney Int 2005;68:14-22.

36. Heyman SN, Rosenberger C, Rosen S. Regional alterations in renal haemodynamics and oxygenation: a role in contrast medium-induced nephropathy. Nephrol Dial Transplant 2005;20 Suppl 1:i6-11.

37. Molitoris BA, Dahl R, Geerdes A. Cytoskeleton disruption and apical redistribution of proximal tubule $\mathrm{Na}(+)-\mathrm{K}(+)$ ATPase during ischemia. Am J Physiol 1992;263:F488-95.

38. Bellomo R, Kellum JA, Ronco C, et al. Acute kidney injury in sepsis. Intensive Care Med 2017;43:816-28.

39. Petek BJ, Bravo PE, Kim F, et al. Incidence and risk factors for postcontrast acute kidney injury in survivors of sudden cardiac arrest. Ann Emerg Med 2016;67:469-76.e1.

40. Ostermann M, Joannidis M. Acute kidney injury 2016: diagnosis and diagnostic workup. Crit Care 2016;20:299. 